\title{
@creative
commons
}

\section{Extending patches to fullerenes}

\author{
Christina Graves \\ The University of Texas at Tyler, Department of Mathematics \\ Tyler, TX 75799, USA \\ Jennifer McLoud-Mann \\ University of Washington Bothell, School of STEM \\ Bothell, WA 98011, USA \\ Kristen Stagg Rovira \\ The University of Texas at Tyler, Department of Mathematics \\ Tyler, TX 75799, USA
}

Received 10 May 2013, accepted 7 June 2014, published online 8 December 2014

\begin{abstract}
In this paper we consider fullerene patches that can be extended to pseudconvex patches. We show that all fullerene disks with three or fewer pentagons can be extended to pseudoconvex patches, and that all pseudoconvex patches can be extended to fullerenes.
\end{abstract}

Keywords: Fullerenes, fullerene patches, boundary codes, pseudoconvex patches.

Math. Subj. Class.: 05C10, 05C75, 92 E10

\section{Preliminaries}

A fullerene is a trivalent planar graph whose faces consist solely of hexagons and pentagons. A fullerene patch, or patch, is similar; it is a planar graph where all faces are hexagons and pentagons except one outer face, with vertices not on the outer face having degree 3 and vertices on the outer face having degree 2 or 3 . An easy way to create a fullerene patch from a fullerene is to trace a closed circuit on a fullerene and delete all faces on one side of the circuit. However, it is not so easy to create a fullerene from a fullerene patch. In fact, there are many fullerene patches that cannot be extended to a fullerene as we will see later. We do find a family of patches that can be extended to fullerenes.

E-mail addresses: cgraves@uttyler.edu (Christina Graves), jmcloud@uw.edu (Jennifer McLoud-Mann), kstagg@uttyler.edu (Kristen Stagg Rovira)

(a) (i) This work is licensed under http://creativecommons.org/licenses/by/3.0/ 

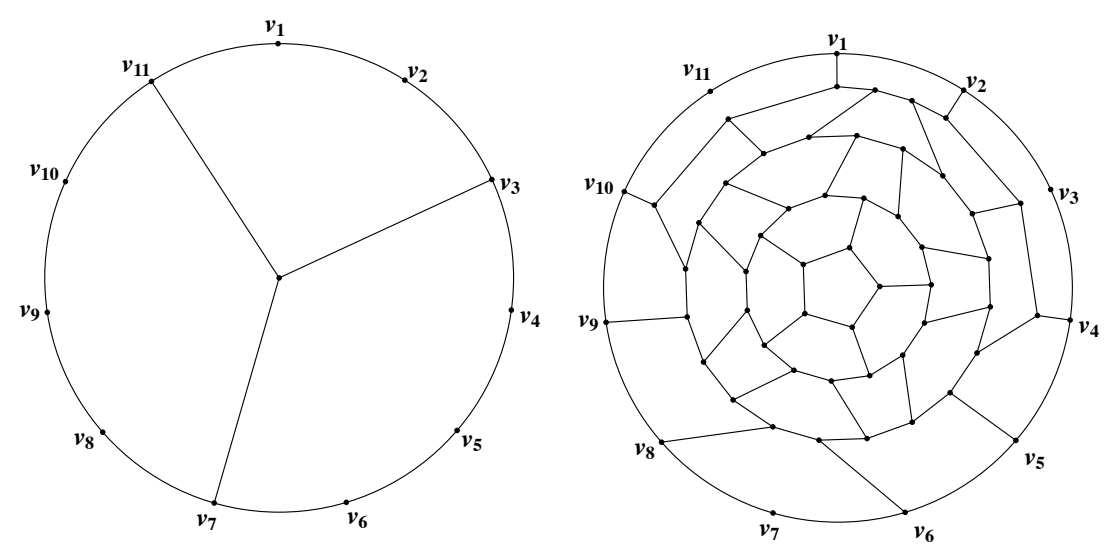

Figure 1: A patch and its complement. The patch on the left has boundary code $223(2223)^{2}$ and side parameters $[1,0,1,0,1]$ and the patch on the right has boundary code $332(3332)^{2}$.

An obvious characteristic of interest of a fullerene patch is the boundary. The boundary code of a patch is a sequence of 2's and 3's corresponding to the valences of the vertices on the outer face listed in cyclic order. It does not matter which vertex we start with nor which direction we travel around the patch; hence we make no distinction between a boundary code and its cyclic permutations or inverse. Given a boundary code $\mathcal{S}$, the complement of $\mathcal{S}$, denoted $\mathcal{S}^{c}$ is a sequence of 2's and 3's with a 2 every place $\mathcal{S}$ has a 3, and a 3 every place $\mathcal{S}$ has a 2 .

To determine if a fullerene patch extends to a fullerene, we need to consider the following question: Given a fullerene patch $\Pi$ with boundary code $\mathcal{S}$, does there exist a fullerene patch $\Pi^{c}$ with boundary code $\mathcal{S}^{c}$ ? If $\Pi^{c}$ exists, we can identify the vertices and edges on the boundary of $\Pi$ with the corresponding vertices and edges on the boundary of $\Pi^{c}$ to get a fullerene (see Figure 1).

The general question of interest is known in the literature as the PentHex Puzzle: Given a sequence of 2's and 3's, does there exist a fullerene patch with that sequence as its boundary code? Some variations on this question were explored in [3], [1], [6], and [4] among other places.

For large patches, the boundary code can be unwieldy to work with. Generalizing the definitions from [8], [7], [5] and [2], we define the following.

\section{Definition 1.1.}

1. A break edge is an edge on the boundary whose endpoints are both of degree two.

2. A bend edge is an edge on the boundary whose endpoints are both of degree three.

3. A side of a patch is a path on the boundary between a consecutive pair of break edges, including the break edges. The length of a side is the number of degree three vertices on the side. 
4. A straight side is a side with no bend edges.

5. A bent side is a side containing at least one bend edge. A straight segment of a bent side is a subpath of a bent side between either a break edge and the closest bend edge, or between two consecutive bend edges.

We can now think of the boundary of a patch as sections of straight sides and straight segments connected by break edges or bend edges. A patch with no bend edges, called a pseudoconvex patch, has only straight sides and thus has boundary code $2(23)^{\ell_{1}} 2(23)^{\ell_{2}} \cdots 2(23)^{\ell_{s}}$. Rather than writing a sequence of 2's and 3's to describe our boundary, we describe the boundary by the lengths of the straight sides $\left[\ell_{1}, \ell_{2}, \ldots, \ell_{s}\right]$, called side parameters of the patch.

If the patch has bend edges, we would still like to consolidate the information found in the boundary code. A bent side with consecutive straight segments of lengths $a_{1}, a_{2}, \ldots a_{t}$ can be described by $\left(a_{1}, \ldots, a_{t}\right)$. Thus, a patch with five straight sides of length 5 followed by a bent side with five straight segments of length 1 (see Figure 3 ) can be described by the side parameters $[5,5,5,5,5,(1,1,1,1,1)]$.

A patch with no break edges has zero sides and thus cannot be described by side parameters. If such a patch also has no bend edges, then we describe it by its boundary code $(23)^{\ell_{0}}$ where $\ell_{0}$ is the number of faces on the boundary. If a patch has no break edges but does have bend edges, then we describe it by its boundary code $3(32)^{a_{1}-1} 3(32)^{a_{2}-1} \cdots 3(32)^{a_{t}-1}$ where $a_{1}, a_{2}, \ldots, a_{t}$ represent the lengths of the straight segments in cyclic order.

The advantage of describing a patch by its side parameters rather than its boundary code is that this notation makes it effortless to find the number of sides (and hence break edges) of a patch. In fact, we can also tell exactly how many pentagons a patch must have by using Euler's formula.

Lemma 1.2. In a fullerene patch, the number of break edges $s$, the number of bend edges $e$, and the number of pentagons $p$ are related by

$$
p=6-s+e
$$

Proof. It is well-known (see for instance [1]) that the number of pentagons in a patch is equal to $6-d_{2}+d_{3}$ where $d_{i}$ is the number of degree $i$ vertices on the boundary. By definition, the number of degree 2 vertices and the number of degree three vertices on the boundary are the same except on a break edge or a bend edge. Each break edge increases the number of degree 2 vertices by 1 , and each bend edge increases the number of degree 3 vertices by 1 .

In this paper, we investigate two specific types of fullerene patches: pseudoconvex patches and fullerene disks or simply disks. A disk of radius $r$ is a fullerene patch with a central face, and every face on the boundary is distance (measured as graph distance in the dual) exactly $r$ from the central face.

Our ultimate goal is to find a family of patches that can be extended to fullerenes. We begin by showing that a pseudoconvex patch can be extended to a fullerene. We then show that all disks with three or fewer pentagons can be extended to fullerenes. 


\section{Pseudoconvex Patches}

Because a pseudoconvex patch has no bend edges, its boundary can be described by its side parameters $\left[\ell_{1}, \cdots, \ell_{s}\right]$ if it has $s \geq 1$ sides or by the boundary code $(23)^{\ell_{0}}$ if it has zero sides. The relationships between the lengths of these sides were explored in detail and summarized in Theorem 3.6 of [8]. We include a weaker lemma here.

Lemma 2.1. The side parameters $\left[\ell_{1}, \cdots, \ell_{s}\right]$ of a pseudoconvex patch, excluding the patch with side parameters $[0,0,0,0,0]$, satisfy the inequality $\ell_{1}+\cdots+\ell_{s} \geq 6-s$ for $1 \leq s \leq 6$.

Proof. If $s=1$ or $s=2$, the result follows directly from [8]. If $s=3$, the side parameters are not of the form $[0,1, k]$ or $[0,0, k]$ for $k \geq 0$ so the result holds. In the case with $s=4$ sides, at least two of the side lengths are non-zero and the result holds. Finally, if $s=5$ or $s=6$, all parameters are nonnegative so the sum is nonnegative. Thus the inequality is satisfied except in the patch consisting of a single pentagon with side parameters $[0,0,0,0,0]$.

To extend a pseudoconvex patch to a fullerene, we need to find a complement for each pseudoconvex patch. The following lemma gives a constructive method for finding the complement patch.

\section{Lemma 2.2. There exists a patch with boundary code}

$$
3(32)^{\ell_{1}} 3(32)^{\ell_{2}} \cdots 3(32)^{\ell_{s}}
$$

if $\ell_{1}+\ell_{2}+\cdots+\ell_{s} \geq 6-s$ and $1 \leq s \leq 6$.

Proof. Start with the patch having boundary code $2(23)^{5}$ (i.e. one side of length 5) as shown in Figure 2. Add $\ell_{1}+\ell_{2}+\cdots+\ell_{s}+s-6$ rings of hexagons to this patch to create a patch with side parameters $\left[\ell_{1}+\cdots+\ell_{s}+s-1\right]$. Next, add a pentagon to the break edge and add hexagons on the boundary everywhere else to yield a patch with boundary code $(23)^{\ell_{1}+\cdots+\ell_{s}+s}$.

We now add hexagons and pentagons to the boundary in the following way. First put a pentagon somewhere on the boundary. Moving clockwise around the boundary, place $\ell_{1}$ hexagons followed by a pentagon; then place $\ell_{2}$ hexagons followed by a pentagon, and continue this process until the patch has a completely new outer ring of hexagons and pentagons (see Figure 2). This constructed patch has the desired boundary.

Theorem 2.3. All pseudoconvex patches can be extended to fullerenes.

Proof. For a pseudoconvex patch consisting of one pentagon with side parameters $[0,0,0,0,0]$, we use a stereographic projection of the dodecahedron onto the plane as the complement patch. For a pseudoconvex patch having side parameters $\left[\ell_{1}, \ldots, \ell_{s}\right]$ satisfying the condition

$$
\ell_{1}+\cdots+\ell_{s} \geq 6-s,
$$

we create the complement patch with boundary code $3(32)^{\ell_{1}} 3(32)^{\ell_{2}} \cdots 3(32)^{\ell_{s}}$ as described in Lemma 2.2 and identify the boundaries to create a fullerene. Given a pseudoconvex patch with no sides and boundary code $(23)^{\ell_{0}}$, we create a second patch $(32)^{\ell_{0}}$ identical to the first and then identify corresponding edges and vertices appropriately to create a fullerene. 

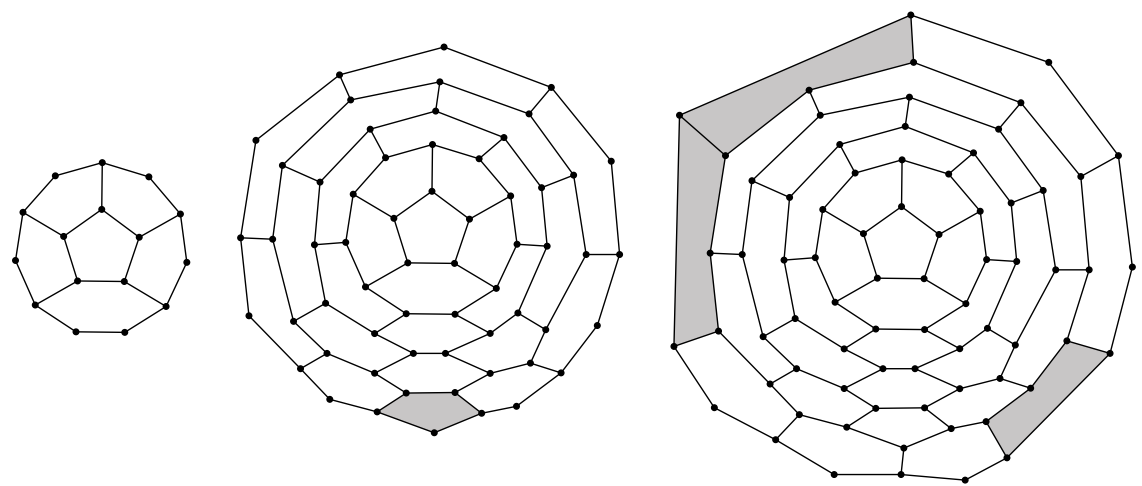

Figure 2: Creating a patch with boundary code $3(32)^{2} 3(32)^{3} 3(32)^{0}$. The $2(23)^{5}$ patch is shown on the left, the $(23)^{8}$ patch in the middle, and the desired patch on the right.

\section{Disks}

Because pseudoconvex patches extend to fullerenes, a patch that extends to a pseudoconvex patch also extends to a fullerene. One type of patch to explore is a fullerene disk. We will show that all disks with three or fewer pentagons extend to pseudoconvex patches; however, a disk with four or more pentagons may not extend to a fullerene. Consider a disk with four pentagons having side parameters $[5,5,5,5,5,(1,1,1,1,1)]$ as shown in Figure 3 . If this patch could be extended to a fullerene, we would need to place a face adjacent to the four shaded pentagons. Such a face would have to have at least seven edges which is not allowed in a fullerene. Thus we restrict our attention to disks with three or less pentagons.

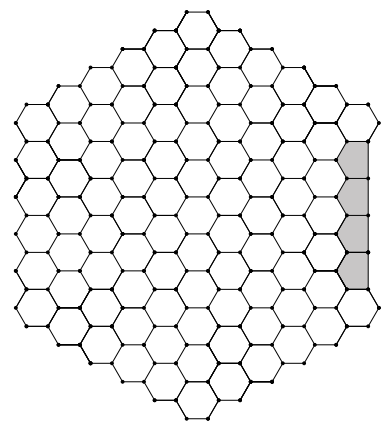

Figure 3: A disk with side parameters $[5,5,5,5,5,(1,1,1,1,1)]$. This patch cannot be extended to a fullerene.

Disks are a nice family of patches to study because all disks can be constructed by repeatedly adding layers of faces to a smaller disk. A layer is a collection of faces placed on the boundary of a disk of a radius $r$ so that each new face is distance $r+1$ from the 

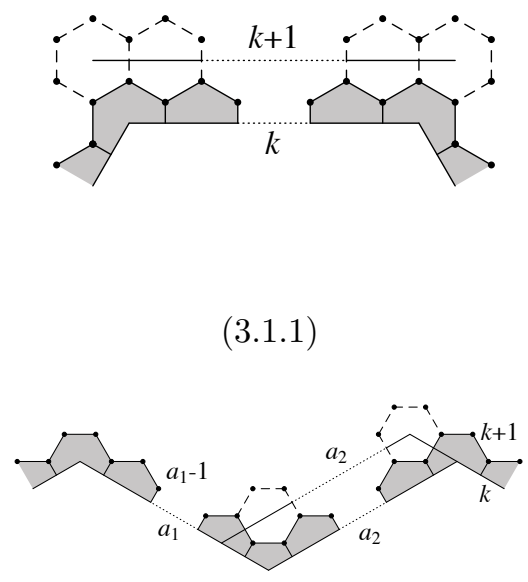

(3.1.3)

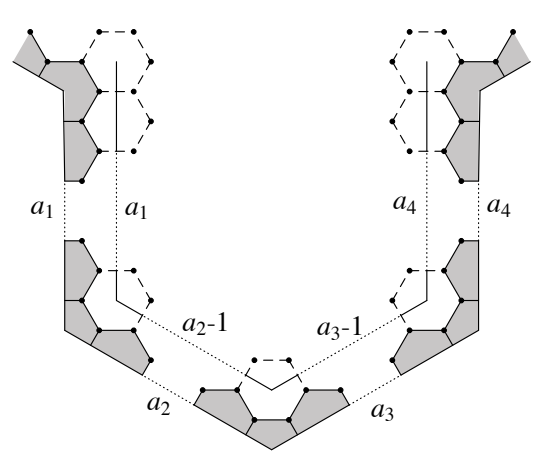

(3.1.2)

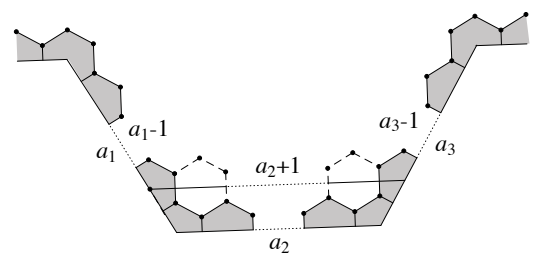

(3.1.4)

Figure 4: Depiction of Lemma 3.1 and how adding hexagons changes side parameters.

central face and and no faces from the original disk are on the new boundary. The word layer can be slightly misleading, though, because there can be faces added which are not on the boundary of the new patch. Figures 5, 6, and 7 show partial patches with one layer added.

Because many of the remaining arguments involve adding layers of hexagons to existing patches, it is convenient to discuss how such an action affects the side parameters. Adding a row of hexagons to a side of a patch means that each edge of the original boundary is incident with a newly added hexagon. Figure 4 and Lemma 3.1 demonstrate this action.

\section{Lemma 3.1.}

3.1.1. Adding a row of hexagons to the boundary of a straight side increases its length by 1.

3.1.2. Adding a partial layer of hexagons to a bent side with middle parameters larger than 1 keeps the outer parameters the same and decreases the middle parameters by 1 .

3.1.3. Adding a row of hexagons to a straight segment $a_{2}$ of the bent side $\left(a_{1}, a_{2}\right)$ with $a_{1}>1$, not including the break edge, decreases $a_{1}$ by 1 , keeps $a_{2}$ the same, and increases the side following $a_{2}$ by 1 .

3.1.4. Adding a row of hexagons to the straight segment $a_{2}$ of a bent side $\left(a_{1}, a_{2}, a_{3}\right)$ with $a_{1}, a_{3}>1$, decreases $a_{1}$ and $a_{3}$ by 1 and increases $a_{2}$ by 1 . 
The lemma tells us how the side parameters of a patch change when we add a layer of hexagons for almost all situations. We will consider special cases for adding a layer of hexagons for patches having a bent side containing a middle parameter equal to one in later proofs.

The next three lemmas give the side parameters for all disks with three or fewer pentagons.

Lemma 3.2. A disk with at most one pentagon has side parameters satisfying one of the following:

A1. $\left[\ell_{1}, \ell_{2}, \ell_{3}, \ell_{4}, \ell_{5}, \ell_{6}\right]$

B1. $\left[\ell_{1}, \ell_{2}, \ell_{3}, \ell_{4}, \ell_{5}\right]$

B2. $\left[\ell_{1}, \ell_{2}, \ell_{3}, \ell_{4}, \ell_{5},\left(a_{1}, a_{2}\right)\right]$

Proof. A disk consisting only of hexagons has the form $[r, r, r, r, r, r]$. For a disk containing one pentagon on the boundary, there are two cases. Either the disk consists of only one face, a pentagon, and has side parameters $[0,0,0,0,0]$, or the disk can be constructed by adding faces to a disk containing no pentagons. Starting with a disk satisfying condition $A 1$, adding a layer of faces with a pentagon on a break edge and hexagons everywhere else yields a $B 1$ patch, and adding a layer with a pentagon on a straight side yields a $B 2$ patch.

If a disk with one pentagon does not have its pentagon on the boundary, then the patch can be viewed as a disk containing one pentagon on the boundary with layers of hexagons added to it. Using Lemma 3.1, adding layers of hexagons to a $B 1$ or $B 2$ patch yields a $B 1$ or $B 2$ patch respectively.

Lemma 3.3. A disk with two pentagons has side parameters satisfying one of the following:

C1. $\left[\ell_{1}, \ell_{2}, \ell_{3}, \ell_{4}\right]$

C2. $\left[\ell_{1}, \ell_{2}, \ell_{3}, \ell_{4},\left(a_{1}, a_{2}\right)\right]$

C3. $\left[\ell_{1}, \ell_{2}, \ell_{3}, \ell_{4},\left(a_{1}, a_{2}\right),\left(b_{1}, b_{2}\right)\right]$

C4. $\left[\ell_{1}, \ell_{2}, \ell_{3},\left(a_{1}, a_{2}\right), \ell_{4},\left(b_{1}, b_{2}\right)\right]$

C5. $\left[\ell_{1}, \ell_{2},\left(a_{1}, a_{2}\right), \ell_{3}, \ell_{4},\left(b_{1}, b_{2}\right)\right]$

C6. $\left[\ell_{1}, \ell_{2}, \ell_{3}, \ell_{4}, \ell_{5},\left(a_{1}, a_{2}, a_{3}\right)\right]$

Proof. We start by considering disks containing two pentagons with different distances from the central face, and the pentagon farthest from the central face on the boundary. Starting with a disk containing one pentagon satisfying condition $B 1$, adding a layer of faces with a pentagon on a break edge yields a $C 1$ patch, and adding a layer with a pentagon on a straight side would yield a $C 2$ patch. Starting with a disk containing one pentagon satisfying condition $B 2$, adding a layer of faces with a pentagon on a break edge results in a $C 2$ patch, adding a layer with a pentagon on a straight side yields a $C 3, C 4$, or $C 5$ patch, and adding a layer with a pentagon on a straight segment of the bent side would yield a $C 6$ patch. 


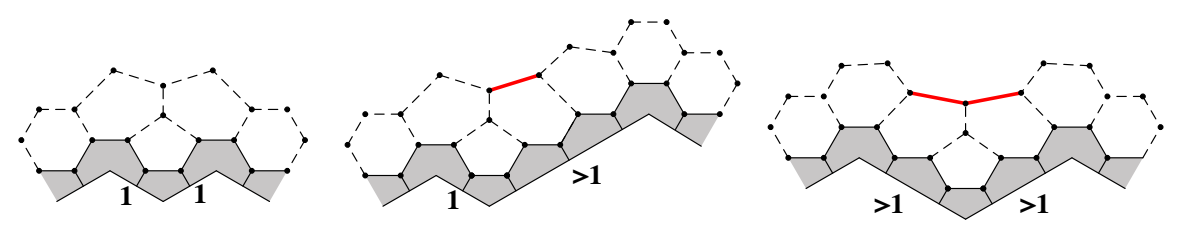

Figure 5: Adding a pentagon to a bend edge. The red edges are bend edges in the new patch.

When adding a layer of faces with a pentagon on the bend edge of a $B 2$ patch, there are three cases to consider. If $a_{1}=a_{2}=1$, the resulting patch is a $C 1$ patch. If $1=a_{1}<a_{2}$, the new patch is a $C 2$ patch. Lastly, if $a_{1}, a_{2}>1$, the resulting patch is a $C 6$ patch, with the middle component of the bent edge having length 1 (see Figure 5).

Now consider disks where both pentagons are the same distance from the central face and are both on the boundary. There are no disks of radius 0 containing two pentagons, so we can construct these patches by starting with a disk containing no pentagons and adding the pentagons to the same layer. Starting with a patch with no pentagons satisfying condition $A 1$, adding a layer with two pentagons on two different break edges yields a $C 1$ patch, adding a layer with one pentagon on a break edge and one on a straight side would result in a $C 2$ patch, adding a layer with two pentagons on two different straight sides would yield a $C 3, C 4$, or $C 5$ patch, and adding a layer with two pentagons on the same straight side would result in a $C 6$ patch.

Notice every disk we have constructed has a pentagon on the boundary. To construct disks with two pentagons neither of which is on the boundary, we simply construct a disk with two pentagons at least one of which is on the boundary, and add layers of hexagons. By Lemma 3.1, adding layers of hexagons to a $C i$ patch yields a $C i$ patch for $1 \leq i \leq 5$. When considering a $C 6$ patch, we assume without loss of generality that $a_{1} \leq a_{3}$. Adding less than $a_{2}+a_{1}-1$ layers of hexagons to a $C 6$ patch results in a $C 6$ patch, and adding at least $a_{2}+a_{1}-1$ layers of hexagons to a $C 6$ patch would yield a $C 1$ or $C 2$ patch. The $C 1$ patch results if $a_{1}=a_{3}$ and the $C 2$ patch results otherwise.

Lemma 3.4. A disk with three pentagons has side parameters satisfying one of the following:

D1. $\left[\ell_{1}, \ell_{2}, \ell_{3}\right]$

D2. $\left[\ell_{1}, \ell_{2}, \ell_{3},\left(a_{1}, a_{2}\right)\right]$

D3. $\left[\ell_{1}, \ell_{2}, \ell_{3},\left(a_{1}, a_{2}\right),\left(b_{1}, b_{2}\right)\right]$

D4. $\left[\ell_{1}, \ell_{2},\left(a_{1}, a_{2}\right), \ell_{3},\left(b_{1}, b_{2}\right)\right]$

D5. $\left[\ell_{1}, \ell_{2}, \ell_{3},\left(a_{1}, a_{2}\right),\left(b_{1}, b_{2}\right),\left(c_{1}, c_{2}\right)\right]$

D6. $\left[\ell_{1}, \ell_{2},\left(a_{1}, a_{2}\right), \ell_{3},\left(b_{1}, b_{2}\right),\left(c_{1}, c_{2}\right)\right]$

D7. $\left[\ell_{1},\left(a_{1}, a_{2}\right), \ell_{2},\left(b_{1}, b_{2}\right), \ell_{3},\left(c_{1}, c_{2}\right)\right]$ 
D8. $\left[\ell_{1}, \ell_{2}, \ell_{3}, \ell_{4},\left(a_{1}, a_{2}, a_{3}\right)\right]$

D9. $\left[\ell_{1}, \ell_{2}, \ell_{3}, \ell_{4},\left(a_{1}, a_{2}, a_{3}\right),\left(b_{1}, b_{2}\right)\right]$

D10. $\left[\ell_{1}, \ell_{2}, \ell_{3},\left(a_{1}, a_{2}, a_{3}\right), \ell_{4},\left(b_{1}, b_{2}\right)\right]$

D11. $\left[\ell_{1}, \ell_{2},\left(a_{1}, a_{2}, a_{3}\right), \ell_{3}, \ell_{4},\left(b_{1}, b_{2}\right)\right]$

D12. $\left[\ell_{1}, \ell_{2}, \ell_{3},\left(a_{1}, a_{2}, a_{3}, a_{4}\right)\right]$

Proof. We begin by showing that that all disks with three pentagons having at least one pentagon on the boundary satisfy the criteria given. To show that all three-pentagon disks have the listed side parameters, we add layers of hexagons to three-pentagon disks with a pentagon on the boundary.

First, consider a three-pentagon disk with exactly one pentagon on the boundary. Such disks are created by starting with a two-pentagon disk and adding a layer of faces containing exactly one pentagon. Starting with a $C 1$ patch, adding a layer of faces with a pentagon on a break edge yields a $D 1$ patch, and adding a layer with a pentagon on a straight side yields a $D 2$ patch. Starting with a $C 2$ patch, adding a layer of faces with a pentagon on a break edge yields a $D 2$ patch, and adding a layer of faces with a pentagon on a straight side yields a $D 3$ or $D 4$ patch. If a layer of faces is added to a $C 2$ patch with a pentagon on a bend edge, then (following the proof of Lemma 3.3) a $D 1, D 2$, or $D 8$ patch is obtained. If a layer is added with a pentagon on a straight straight segment of a bent side of a $C 2$ patch, the resulting disk is a $D 8$ patch.

Starting with a $C 3-C 5$ patch, adding a layer of faces with a pentagon on a straight side yields a $D 5-D 7$ patch, and adding a layer with a pentagon on a straight segment of a bent side yields a $D 9, D 10$, or $D 11$ patch. Adding a layer of faces with a pentagon on a break edge of a $C 3$ patch yields a $D 3, D 4$, or $D 8$ patch, with a $D 8$ patch occurring if the break edge is between the two bent sides. Adding a layer with a pentagon on a break edge of a $C 4$ or $C 5$ patch yields a $D 3$ or $D 4$ patch. Finally, adding a layer of faces with a pentagon on a bend edge on a $C 3-C 5$ patch yields a $D 2, D 3, D 4, D 9, D 10$, or $D 11$ patch.

The last case involves adding a layer of faces with exactly one pentagon to a $C 6$ patch. Adding a layer of faces with a pentagon on a break edge results in a $D 8$ patch, adding a layer with a pentagon on a straight side yields a $D 8-D 11$ patch, and adding a layer with a pentagon on a straight segment of a bent side results in a $D 12$ patch. When adding a layer of faces with a pentagon on a bend edge of a $C 6$ patch there are a few cases to consider. Without loss of generality, assume that the pentagon is added to the bend edge between the straight segment of length $a_{1}$ and the straight segment of length $a_{2}$. If $a_{1}>1$ and $a_{2}>2$ the resulting patch is a $D 12$ patch with the new bent side having lengths $\left(a_{1}-1,1, a_{2}-2, a_{3}\right)$ (see Figure 6 ). In the cases were either $a_{1}=1$ or $a_{2} \in\{1,2\}$, some care needs to be taken. In these cases, the length of $a_{3}$ can affect the type of new patch; however, in every case, the resulting patch is a $D 2$ or $D 8$ patch. Figure 7 shows these cases in full detail.

Now consider the three-pentagon disks with exactly two pentagons on the boundary. To create such disks, we start with either a $B 1$ or $B 2$ patch. Adding a layer of faces with two pentagons to a $B 1$ patch is very similar to adding two pentagons to an $A 1$ patch, which was explored in the proof of Lemma 3.3. Thus adding a layer with two pentagons on a $B 1$ patch results in a $D 1-D 4$ or $D 8$ patch. Using similar arguments as before, adding a layer 


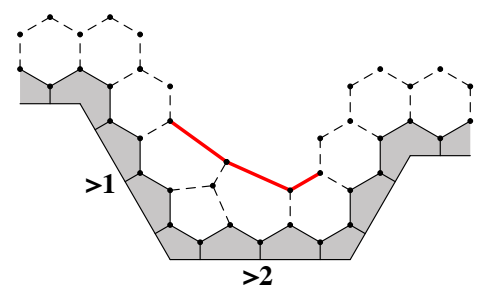

Figure 6: Adding a pentagon to a bend edge in a $C 6$ patch. The red edges are bend edges in the new patch.
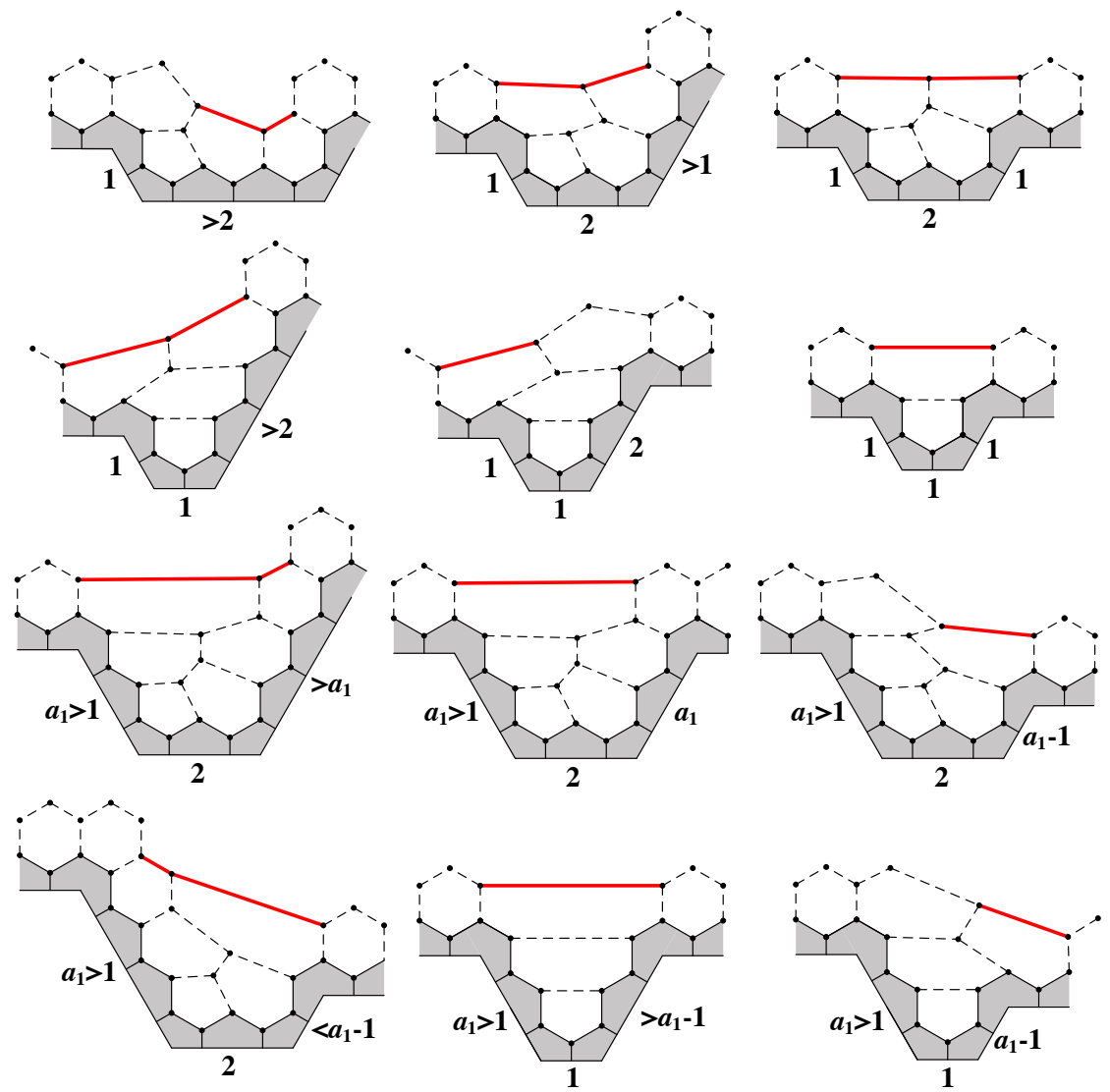

Figure 7: Adding a pentagon to a bend edge in a $C 6$ patch. The red edges are bend edges in the new patch. 
with two pentagons to a $B 2$ patch results in a $D 2-D 12$ patch if neither pentagon is on a bend edge. Adding a layer with one of the pentagons on a bend edge requires the cases seen in Figure 5. Adding a layer with one pentagon on the bend edge and one pentagon on a break edge yields a $D 1, D 2$, or $D 8$ patch. Adding a layer with one pentagon on the bend edge and one pentagon on a straight side results in a $D 2-D 4$ or $D 9-D 11$ patch. Finally, adding a layer with one pentagon on the bend edge and one pentagon on a straight segment of the bent side (which can only be done if the straight segment is greater than 1) results in a $D 8$ patch.

Three-pentagon disks with all three pentagons on the boundary are formed by adding a layer to a disk of hexagons. Starting with an $A 1$ patch, adding a layer with three pentagons results in a $D 1-D 11$ patch using similar arguments to those above.

Notice every patch we have constructed has a pentagon on the boundary. For those that do not, we simply construct a previous patch and add layers of hexagons. By Lemma 3.1, adding layer of hexagons to a $D i$ patch yields a $D i$ patch for $1 \leq i \leq 7$. For disks that have a bent side with at least three straight segments, the resulting patch's side parameters differ based on the number of layers added. For instance, adding one layer of hexagons to a patch with the side parameters $\left[\ell_{1}, \ell_{2}, \ell_{3}, \ell_{4},\left(a_{1}, a_{2}, a_{3}\right)\right]$ and $a_{2}>1$ results in a patch with side parameters $\left[\ell_{1}+1, \ell_{2}+1, \ell_{3}+1, \ell_{4}+1,\left(a_{1}, a_{2}-1, a_{3}\right)\right]$. Furthermore, adding a layer of hexagons to a patch with side parameters $\left[\ell_{1}, \ell_{2}, \ell_{3}, \ell_{4},\left(a_{1}, 1, a_{3}\right)\right]$ yields a patch with side parameters $\left[\ell_{1}+1, \ell_{2}+1, \ell_{3}+1, \ell_{4}+1,\left(a_{1}-1,1, a_{3}-1\right)\right]$. Thus, adding less than $a_{2}+\min \left\{a_{1}, a_{3}\right\}-1$ rings to a $D j$ patch will yield a $D j$ patch for $8 \leq j \leq 11$.

Adding at least $a_{2}+\min \left\{a_{1}, a_{3}\right\}-1$ rings to a $D 8$ patch yields a $D 1$ patch if $a_{1}=a_{3}$ or $D 2$ patch otherwise. Adding at least $a_{2}+\min \left\{a_{1}, a_{3}\right\}-1$ rings to a $D 9$ patch yields a $D 2$ patch if $a_{1}=a_{3}$, a $D 3$ patch if $a_{1}<a_{3}$, or a $D 8$ patch if $a_{1}>a_{3}$. Adding at least $a_{2}+\min \left\{a_{1}, a_{3}\right\}-1$ rings to a $D 10$ or $D 11$ patch yields a $D 2$ patch if $a_{1}=a_{3}$ or $D 4$ patch otherwise.

Now let us consider adding rings of hexagons to a $D 12$ patch; without loss of generality assume $a_{2} \leq a_{3}$. Adding less than $a_{2}+\min \left\{a_{1}-1,\left\lfloor\frac{a_{3}-a_{2}}{2}\right\rfloor\right\}$ rings yields another $D 12$ patch. Adding exactly $a_{2}+\min \left\{a_{1}-1,\left\lfloor\frac{a_{3}-a_{2}}{2}\right\rfloor\right\}$ rings yields a $D 1, D 2$, or $D 8$ patch. From arguments above, adding further rings of hexagons gives a $D 1, D 2$, or $D 8$ patch.

Now that we have shown the side parameters of disks with three or fewer pentagons fall into different classes, we show that any patch with these side parameters can be extended to a pseudoconvex patch.

Lemma 3.5. Any patch with one of the following descriptions of its side parameters can be extended to a pseudoconvex patch:

1. $\left[\ell_{1}, \ell_{2}, \ldots, \ell_{s}\right]$

2. $\left[\ell_{1}, \ell_{2}, \ldots, \ell_{s},\left(a_{1}, a_{2}\right)\right]$

3. $\left[\ell_{1}, \ell_{2}, \ldots, \ell_{s},\left(a_{1}, a_{2}\right),\left(b_{1}, b_{2}\right)\right]$

4. $\left[\ell_{1}, \ell_{2}, \ldots, \ell_{s},\left(a_{1}, a_{2}\right), \ell_{s+1}, \ldots, \ell_{t},\left(b_{1}, b_{2}\right)\right]$

5. $\left[\ell_{1}, \ell_{2}, \ldots, \ell_{s},\left(a_{1}, a_{2}\right),\left(b_{1}, b_{2}\right),\left(c_{1}, c_{2}\right)\right]$

6. $\left[\ell_{1}, \ell_{2}, \ldots, \ell_{s},\left(a_{1}, a_{2}\right),\left(b_{1}, b_{2}\right), \ell_{s+1}, \ldots, \ell_{t},\left(c_{1}, c_{2}\right)\right]$

7. $\left[\ell_{1}, \ell_{2}, \ldots, \ell_{s},\left(a_{1}, a_{2}\right), \ell_{s+1}, \ldots, \ell_{t},\left(b_{1}, b_{2}\right), \ell_{t+1}, \ldots, \ell_{u},\left(c_{1}, c_{2}\right)\right]$ 

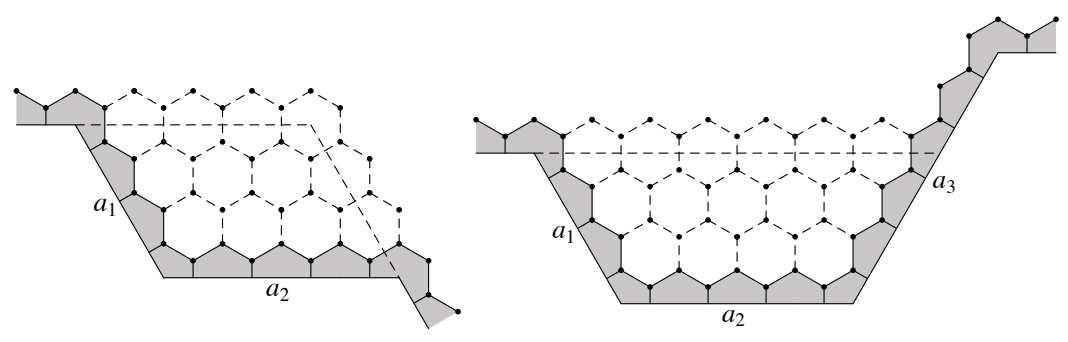

Figure 8: Filling in the bent side $\left(a_{1}, a_{2}\right)$ and partially filling in the bent side $\left(a_{1}, a_{2}, a_{3}\right)$.

8. $\left[\ell_{1}, \ell_{2}, \ldots, \ell_{s},\left(a_{1}, a_{2}, a_{3}\right)\right]$

9. $\left[\ell_{1}, \ell_{2}, \ldots, \ell_{s},\left(a_{1}, a_{2}\right),\left(b_{1}, b_{2}, b_{3}\right)\right]$

10. $\left[\ell_{1}, \ell_{2}, \ldots, \ell_{s},\left(a_{1}, a_{2}\right), \ell_{s+1}, \ldots, \ell_{t},\left(b_{1}, b_{2}, b_{3}\right)\right]$

11. $\left[\ell_{1}, \ell_{2}, \ldots, \ell_{s},\left(a_{1}, a_{2}, a_{3}, a_{4}\right)\right]$.

Proof. Let $\Pi$ be a patch with one of the following descriptions above. If $\Pi=\left[\ell_{1}, \ell_{2}, \ldots\right.$, $\left.\ell_{s}\right]$, then it has no bend edges and is pseudoconvex. If $\Pi=\left[\ell_{1}, \ell_{2}, \ldots, \ell_{s},\left(a_{1}, a_{2}\right)\right]$, then adding $a_{1}$ rows of $a_{2}$ hexagons to the straight segment $a_{2}$, not including the break edge, yields the pseudoconvex patch $\left[\ell_{1}+a_{1}, \ell_{2}, \ldots, \ell_{s-1}, \ell_{s}+a_{2}\right]$ by Lemma 3.1 (see Figure 8).

If the side parameters of $\Pi$ are of the form $\left[\ell_{1}, \ell_{2}, \ldots, \ell_{s},\left(a_{1}, a_{2}\right),\left(b_{1}, b_{2}\right)\right]$ or $\left[\ell_{1}, \ell_{2}, \ldots, \ell_{s},\left(a_{1}, a_{2}\right), \ell_{s+1}, \ldots, \ell_{t},\left(b_{1}, b_{2}\right)\right]$, then adding $a_{1}$ rows of $a_{2}$ hexagons to the straight segment $a_{2}$, not including the break edge, yields either

$$
\left[\ell_{1}, \ell_{2}, \ldots, \ell_{s}+a_{2},\left(b_{1}+a_{1}, b_{2}\right)\right]
$$

or

$$
\left[\ell_{1}, \ell_{2}, \ldots, \ell_{s}+a_{2}, \ell_{s+1}+a_{1}, \ldots, \ell_{t},\left(b_{1}, b_{2}\right)\right]
$$

which are both extendable by a previous case. If $\Pi$ has one of the forms

$$
\begin{gathered}
{\left[\ell_{1}, \ell_{2}, \ldots, \ell_{s},\left(a_{1}, a_{2}\right),\left(b_{1}, b_{2}\right),\left(c_{1}, c_{2}\right)\right],} \\
{\left[\ell_{1}, \ell_{2}, \ldots, \ell_{s},\left(a_{1}, a_{2}\right),\left(b_{1}, b_{2}\right), \ell_{s+1}, \ldots, \ell_{t},\left(c_{1}, c_{2}\right)\right],}
\end{gathered}
$$

or

$$
\left[\ell_{1}, \ell_{2}, \ldots, \ell_{s},\left(a_{1}, a_{2}\right), \ell_{s+1}, \ldots, \ell_{t},\left(b_{1}, b_{2}\right), \ell_{t+1}, \ldots, \ell_{u},\left(c_{1}, c_{2}\right)\right],
$$

then adding $a_{1}$ rows of $a_{2}$ hexagons to the straight segment $a_{2}$, not including the break edge, yields a patch with one of the descriptions

$$
\begin{gathered}
{\left[\ell_{1}, \ell_{2}, \ldots, \ell_{s}+a_{2},\left(b_{1}+a_{1}, b_{2}\right),\left(c_{1}, c_{2}\right)\right]} \\
{\left[\ell_{1}, \ell_{2}, \ldots, \ell_{s}+a_{2},\left(b_{1}+a_{1}, b_{2}\right), \ell_{s+1}, \ldots, \ell_{t},\left(c_{1}, c_{2}\right)\right]}
\end{gathered}
$$

or

$$
\left[\ell_{1}, \ell_{2}, \ldots, \ell_{s}+a_{2}, \ell_{s+1}+a_{1}, \ldots, \ell_{t},\left(b_{1}, b_{2}\right), \ell_{t+1}, \ldots, \ell_{u},\left(c_{1}, c_{2}\right)\right]
$$


which are all extendable by previous cases.

Suppose $\Pi=\left[\ell_{1}, \ell_{2}, \ldots, \ell_{s},\left(a_{1}, a_{2}, a_{3}\right)\right]$. Without loss of generality, assume $a_{1} \leq a_{3}$. Adding $a_{1}-1$ rows of hexagons to the $a_{2}$ straight segment results in a patch with parameters

$$
\left[\ell_{1}, \ell_{2}, \ldots, \ell_{s},\left(1, a_{1}+a_{2}-1, a_{3}-a_{1}+1\right)\right]
$$

by Lemma 3.1. Adding one more row yields a patch whose parameters are

$$
\left[\ell_{1}, \ell_{2}, \ldots, \ell_{s-1},\left(\ell_{s}+a_{1}+a_{2}, a_{3}-a_{1}\right)\right]
$$

if $a_{1}<a_{3}$ or

$$
\left[\ell_{s}+a_{1}+a_{2}+\ell_{1}, \ell_{2}, \ell_{3}, \ldots, \ell_{s-1}\right]
$$

if $a_{1}=a_{3}$. Either of these is extendable by a previous case.

If $\Pi$ has the form

$$
\left[\ell_{1}, \ell_{2}, \ldots, \ell_{s},\left(a_{1}, a_{2}\right),\left(b_{1}, b_{2}, b_{3}\right)\right]
$$

or

$$
\left[\ell_{1}, \ell_{2}, \ldots, \ell_{s},\left(a_{1}, a_{2}\right), \ell_{s+1}, \ldots, \ell_{t},\left(b_{1}, b_{2}, b_{3}\right)\right],
$$

then adding $a_{1}$ rows of $a_{2}$ hexagons to the straight segment $a_{2}$, not including the break edge, yields a patch satisfying the form

$$
\left[\ell_{1}, \ell_{2}, \ldots, \ell_{s}+a_{2},\left(b_{1}+a_{1}, b_{2}, b_{3}\right)\right]
$$

or

$$
\left[\ell_{1}, \ell_{2}, \ldots, \ell_{s}+a_{2}, \ell_{s+1}+a_{1}, \ldots, \ell_{t},\left(b_{1}, b_{2}, b_{3}\right)\right]
$$

which are both extendable by previous cases.

Suppose $\Pi=\left[\ell_{1}, \ell_{2}, \ldots, \ell_{s},\left(a_{1}, a_{2}, a_{3}, a_{4}\right)\right]$. Without loss of generality, we may assume that $a_{1} \leq a_{4}$. Note that $a_{1} \leq a_{3}+a_{4}$. We have the following four cases to consider: (i) $0<a_{1}<a_{3}$, (ii) $a_{1}=a_{3}$, (iii) $a_{3}<a_{1}<a_{3}+a_{4}$, and (iv) $a_{1}=a_{3}+a_{4}$. In each of the four cases we will add $a_{1}$ rows of hexagons to the straight segment $a_{2}$ in order to create a patch which is extendable by a previous case.

For (i) and (ii), we begin by adding $a_{1}-1$ rows of hexagons to create a patch with parameters $\left[\ell_{1}, \ell_{2}, \ldots, \ell_{s},\left(1, a_{1}+a_{2}-1, a_{3}-a_{1}+1, a_{4}\right)\right]$ by Lemma 3.1. Adding one more row will yield

$$
\left[\ell_{1}, \ell_{2}, \ldots, \ell_{s-1},\left(\ell_{s}+a_{1}+a_{2}, a_{3}-a_{1}, a_{4}\right)\right]
$$

in case (i) and

$$
\left[\ell_{1}, \ell_{2}, \ldots, \ell_{s-1},\left(\ell_{s}+a_{1}+a_{2}-1,1, a_{4}-1\right)\right]
$$

in case (ii).

For (iii) and (iv), we begin by adding $a_{3}-1$ rows of hexagons to straight side $a_{2}$ to yield $\left[\ell_{1}, \ell_{2}, \ldots, \ell_{s},\left(a_{1}-a_{3}+1, a_{2}+a_{3}-1,1, a_{4}\right)\right]$ by Lemma 3.1. Adding another row yields a $\left[\ell_{1}, \ell_{2}, \ldots, \ell_{s},\left(a_{1}-a_{3}, a_{2}+a_{3}-1,1, a_{4}-1\right)\right]$ patch. For (iii), adding $a_{1}-a_{3}-1$ rows gives

$$
\left[\ell_{1}, \ell_{2}, \ldots, \ell_{s},\left(1, a_{2}+a_{3}-1,1, a_{4}+a_{3}-a_{1}\right)\right]
$$

and finally adding one more row gives a

$$
\left[\ell_{1}, \ell_{2}, \ldots, \ell_{s-1},\left(\ell_{s}+a_{2}+a_{3}-1,1, a_{4}+a_{3}-a_{1}-1\right)\right]
$$




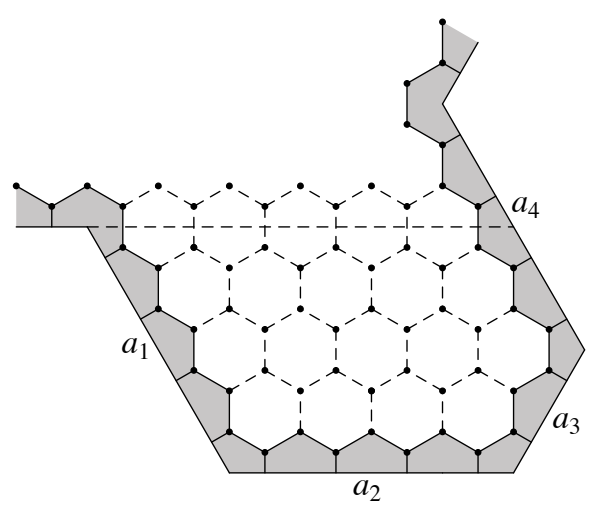

Figure 9: Partially filling in the bent side $\left(a_{1}, a_{2}, a_{3}, a_{4}\right)$.

patch (see Figure 9). For (iv), adding $a_{1}-a_{3}-2$ rows of hexagons gives $\left[\ell_{1}, \ell_{2}, \ldots, \ell_{s},(2\right.$, $\left.\left.a_{2}+a_{3}-1,1,1\right)\right]$, an additional row yields

$$
\left[\ell_{2}, \ell_{3}, \ldots, \ell_{s},\left(1, a_{2}+a_{3}, \ell_{1}\right)\right]
$$

and finally adding another row gives $\left[\ell_{2}, \ell_{3}, \ldots, \ell_{s-1},\left(\ell_{s}+a_{2}+a_{3}, \ell_{1}\right)\right]$.

Theorem 3.6. All disks containing at most three pentagons extend to a fullerene.

Proof. All disks containing at most three pentagons will have side parameters satisfying the conditions of Lemma 3.2, Lemma 3.3, or Lemma 3.4. By Lemma 3.5, these disks can be extended to pseudoconvex patches. By Theorem 2.3, those pseudoconvex patches can be extended to fullerenes.

\section{References}

[1] P. Bonsma and F. Breuer, Finding fullerene patches in polynomial time. In ISAAC 2009, volume 5878 of LNCS (2009), 750-759.

[2] J. Bornhoft, G. Brinkmann and J. Greinus, Pentagon-hexagon-patches with short boundaries, European Journal of Combinatorics 24 (2003), 517-529.

[3] G. Brinkmann and A. W. M. Dress, PentHex puzzles: A reliable and efficient top-down approach to fullerene-structure enumeration, Advance in Applied Math 21 (1998), 473-480.

[4] G. Brinkmann, U.V. Nathusius and A.H.R. Palser, A constructive enumeration of nanotube caps, Discrete Applied Mathematcis 116 (2002), 55-71.

[5] G. Brinkmann and N. Van Cleemput, Classification and Generation of Nanocones, Discrete Applied Mathematics 159 (2011), 1528-1539.

[6] M. Deza, P. W. Fowler, and V. Grishukhin. Allowed boundary sequences for fused polycyclic patches and related algorithmic problems. J. Chem. Inf. Comput. Sci. 41 (2001), 300-308.

[7] J.E. Graver and C. Graves, Fullerene Patches I, Ars Mathematica Contemporanea 3 (2010), $104-120$.

[8] C. Graves and J. McLoud-Mann, Side lengths of pseudoconvex fullerene patches, Ars Mathematica Contemporanea 5 (2012), 291-302. 